\title{
Developments in mobile data system technology - particularly GSM
}

\author{
J. Leske \\ Centre for Telecommunications Information Networking (CTIN) \\ 33 Queen St, Thebarton, SA 5031, Australia \\ Phone +61-8-303 3222, Fax +61-8-303 4405 \\ Email:J.Leske@CTIN.adelaide.edu.au
}

\begin{abstract}
This paper details the current and near future developments in mobile data systems. It looks in particular detail at the work currently being undertaken to provide advanced data capabilities in the GSM digital mobile phone system, particularly packet and high speed circuit switched data. This paper also details other current developments in mobile data systems, from two-way paging systems through to high speed wireless LANs.
\end{abstract}

mobile data, wireless, GSM, GPRS, HSCSD

\section{Keywords}

\section{INTRODUCTION}

According to the Wireless LAN Alliance and the Yankee Group (Chambers, 1996), in North America wireless local area network (WLAN) sales tripled from 1993 to 1995 (US\$157 million). Of the cellular phone users in the USA, approxiamately $2 \%$ use some form of mobile data communications (Data Communications, 1995). In Europe the use of data over cellular phone networks is conservatively forecast to grow to $3 \%$ by 1998 and to $18 \%$ by 2003 (Yankee Group, 1996). Other forms of wireless data access exhibit strong growth, with enthusiastic forecasts for their future.

What are the reasons for such strong growth? Why go Wireless?

Customer expectation and work practices have changed to require responsiveness, immediacy, and accuracy. While half of typical USA mobility is within the company building, the remainder comprises: within a campus area (14\%), in metropolitan area (18\%) within the country $(12 \%)$ or out of the country (1\%) (Pryor, 1996). Staying connected allows access to central information storage at the point of service, for consistency, efficiency and security. The 
resurgence in centralised information storage can be seen in the use of client/server systems and the network computer concept. Typical mobile data applications include: field service; fleet management; public safety; field sales; EFTPOS; and the mobile professional seeking data base access and information sharing.

Wireline remote access can meet some of these needs, especially where daily access or updates are sufficient. For broadband style applications, such as video conferencing, a mobile solution cannot meet the bandwidth demand of $1.5 \mathrm{Mbit} / \mathrm{s}$ or higher. But workgroup applications, such as Lotus Notes, rely on large central information stores where a typical database size is $500 \mathrm{MB}$. A dial-in update will often take half an hour, so continual mobile access to the central database has many advantages.

With the many advantages wireless can offer, why isn't it used more frequently?

\section{What's so hard about not having a connecting wire?}

The choices for wireless connection are InfraRed (IR) or radio. IR is limited by line-of-sight issues and so is restricted to localised coverage. IR requires multiple sources to cover a complex geometry like the inside of a partitioned office, and multiple receivers to be in sight of each terminal unit. Interference problems are fairly minimal, however direct sunlight can saturate receivers. Range is quite limited, typically being $3 \mathrm{~m}$ for high speed $(>1 \mathrm{Mbit} / \mathrm{s})$ and $50 \mathrm{~m}$ for low speed IR systems.

Radio is the best current solution for mobility. With appropriate frequencies radio can operate around corners and penetrate inside most buildings and vehicles. However the electromagnetic spectrum is a limited resource which must be shared between multiple uses (TV, radio, RADAR, etc) and multiple users (subscribers). Interference can be from other radio transmitters nearby on the same system; from transmitters on adjacent channels of a different system; intermodulation results from separate frequencies; or noise such as clock signals from devices not intended to be radio transmitters. Signal attenuation is another difficulty: radio signals have losses proportional to distance squared and distance to the fourth power; buildings and trees cause additional losses; at higher frequencies atmospheric effects such as rain also significantly attenuate the signal. Finally, the multiple paths by which a radio signal travels cause fading, which is worse with high frequencies and motion.

Hence the major limitations in radio are that to increase range higher power is needed, with attendant interference issues; and to increase data rate a larger portion of the limited spectrum is required. These difficulties are continuously being addressed to provide and improve mobile data facilities. The following sections of this paper investigate current systems which offer mobile data. capability.

\section{GLOBAL SYSTEM FOR MOBILES (GSM)}

The development of the second generation (digital) cellular mobile phone system, GSM, commenced in Europe in 1982. Commercial operation of GSM, in the 890 to $960 \mathrm{MHz}$ band, subsequently commenced in 1992.

Using TDMA (Time Division Multiple Access), each $200 \mathrm{kHz}$ channel is divided into 8 timeslots. Primarily designed for voice communications, GSM also supports data, facsimile and short pager-like text messages. 


\subsection{Circuit switched data}

The primary method of computer to computer data communication in GSM at present is the circuit-switched data call. GSM currently supports up to $9600 \mathrm{bit} / \mathrm{s}$ asynchronous or synchronous communication, and can also incorporate RF error correction. The raw RF bit rate is $33.8 \mathrm{kbit} / \mathrm{s}$. Channel overheads reduce this to $22.8 \mathrm{kbit} / \mathrm{s}$. Interleaving and some minor forward error correction designed specifically for voice reduce this to $13 \mathrm{kbit} / \mathrm{s}$. Additional error correction for data transmission reduces the available user data rate to $9.6 \mathrm{kbit} / \mathrm{s}$.

Radio Link Protocol (RLP) is the specialist layer 2 error correction and data burst retransmission scheme for GSM. Without RLP, the residual average bit error rate (BER) from the sole use of forward error correction (FEC) schemes is $10^{-3 *}$. When using RLP, the data stream is divided into blocks of 200 bits, which can be selectively retransmitted if they are corrupted by the radio transmission. This reduces the typical bit error rate to $10^{-6}$. The penalty of achieving this is increased end-to-end delay. Without RLP, GSM introduces a maximum delay in transmission from GSM handset to network connection of $330 \mathrm{~ms}$. With RLP, the delay is dependant on the quality of the radio link at the time, with interference causing retransmissions and hence longer delays.

The GSM specification defines links for data transmission to the telephone network via modem bank, to ISDN, and direct access to X.25. Modem connection has been available since November 1994, and all GSM operators in Australia currently support this. ISDN and X.25 access are to be offered soon.

The data rate of $9.6 \mathrm{kbit} / \mathrm{s}$ may seem slow compared to $28.8 \mathrm{k} \mathrm{kbit} / \mathrm{s}$ now common over the telephone network. Additionally these V.34 modems cannot be used with GSM while they can be used over the analogue cellular network. Because GSM is a fully digital system, instead of plugging a modem into a computer, a "data card" is used instead, which converts the data stream and adds the GSM transmission protocols to it. However V.34 is not suitable for the noisy mobile environment. Typically over the analogue network the reliable throughput obtainable is $2400 \mathrm{bit} / \mathrm{s}$. This is being addressed by special analogue mobile error correction protocols like ETC and MNP-10 introduced last year, which by performing a similar function to RLP bring the reliable throughput up to $9.6 \mathrm{kbit} / \mathrm{s}$, or potentially $14.4 \mathrm{kbit} / \mathrm{s}$ for a stationary phone in a high signal strength area.

Data compression standard V.42bis has been approved for use with GSM. This is currently being implemented in GSM networks around the world. For compressible data, such as text, V.42bis can offer a 2:1 or better improvement in throughput.

GSM data communications are currently used for facsimile services (transmit mostly rather than receive), dial-in access to hosts, for Email, and file access. The limited data rate means that applications like LAN extension or large file transfers (such as multimedia or the World Wide Web) are quite limited over GSM. Additionally, the connection orientated nature means that long duration calls become very expensive. Staying connected to the office LAN all day is unrealistic, and even spending an hour connected to central office while conducting an interview is prohibitive in cost.

Where applications have not been designed for mobile operation, the overhead of data transmission added to the occasional delay of RLP, can make interactive operation frustrating. An Email application which echoes keystrokes onto the screen from the central computer can lag typing by a number of characters.

\footnotetext{
* GSM network design calls for a $12 \mathrm{~dB}$ carrier to interference ratio. Where the quality of the radio link is worse, the error rate will be correspondingly higher.
} 
Applications currently running over GSM circuit switched data include field service, sales, fleet telemetry, Email, and remote host access.

\subsection{Short Message Service (SMS)}

SMS messages are 140 byte data packets sent to and from mobile stations. These messages are sent on control channels, thus allowing simultaneous voice conversation. The intended usage for this facility is notification messaging: pager-like 160 character alphanumeric messages. The messages are sent using a store and forward system via a central SMS centre. This guarantees delivery of messages to turned off or out of range phones once they become available again.

SMS is typically used to provide operator transcribed messages and automatic notification of voicemail. It is also used for limited email, and some telemetry applications. In the UK one application developed is as a data link for police offers sending in their reports from Apple Newtons. Enhancements to the GSM standard will provide for chaining of SMS messages together, allowing full email for example, and message overwriting, which would allow stock quotes or weather reports to be sent regularly without overflowing the message storage.

While the performance of a standard SMS system is not that fast, especially because of the store and forward system, a network can accommodate more than one SMS centre, allowing certain centres to be optimised for use as data links, which makes traffic telemetry and similar real-time applications viable.

\subsection{Packet Data over Signalling (PDS)}

PDS was originally proposed by DeTeMobil (the national German mobile telephone network) to provide a data transport mechanism for real-time traffic telemetry. Research is being conducted by the European Union in a project called 'SOCRATES' to design a comprehensive traffic monitoring, toll paying, and driver assistance network for Europe. As DeTeMobil is heavily involved in this, they proposed a solution where the existing cellular network, common throughout Europe and soon to be covering most motorways, could be used as the communications link.

The data rates required are quite moderate: $2400 \mathrm{bit} / \mathrm{s}$ broadcast downlink to cars, and 100 bit/s uplink from cars to the network. Since GPRS, the fully featured packet data system, was considered to be progressing quite slowly, a simpler solution was proposed explicitly for this purpose. The design borrowed heavily from SMS, while also taking on concepts being developed in GPRS. However the time frame for prototype trials has not been met, and it seems as though PDS while being standardised, will not reach prototype stage and be dropped in favour of GPRS once it becomes available.

\subsection{General Packet Radio Service (GPRS)}

GPRS is the main effort for the development of a general purpose packet data capability for GSM, as opposed to the specific capability which SMS provides. The aim is to provide a capability to handle everything from large numbers of telemetry users with small data packets, through to high speed packet data transmission with low latency for individual users up to speeds of $80 \mathrm{kbit} / \mathrm{s}$.

The discussion for developing GPRS has been underway since 1994 at ETSI (European Telecommunications Standards Institute). The broad standard is expected to be finalised by the 
end of this year, followed by detailed design in 1997, with prototypes developed in 1998 (optimistically). The two main drives for the design of GPRS are the 'SOCRATES' road telemetry project, and the European Railways Organisation (UIC) requirements for a unified railway communications and telemetry system.

The UIC desires a system that will not only provide voice access across Europe for train controllers and drivers, but will also provide the data communications link for centralised train monitoring and control, with a particular emphasis on the planned $500 \mathrm{~km} / \mathrm{h}$ next generation of very fast trains (VFT). In this application GPRS would have to provide $99.9 \%$ confidence maximum message delay of 0.5 seconds for a 250 byte message every 2 seconds. This presents some interesting challenges, which should be able to be met.

The basic design of GPRS is constrained to use, as far as practical, the existing GSM system. The intention is to ease the cost of adding GPRS capability to an existing network, to assist in the widespread introduction of the service. Software changes to existing network infrastructure are achieved by downloads from the central operations and maintenance (OAM) system. Physically going to every BTS to change hardware is an order of magnitude more expensive. GPRS will require additional equipment: intelligent network (IN) style 'GPRS support nodes' (GSN) to manage the packet data sessions, located at the base station controller (BSC) or mobile switch (MSC) level.

In contrast consider the approach necessary for cellular digital packet data (CDPD) in North America. To add packet data to the analogue cellular network an entirely separate network is parasitically added to the AMPS network. This involves significant capital and installation costs, with CDPD radio transmitters and receivers added at every base station. After an expenditure of nearly US\$1billion to set up the capability, there is still some doubt as to the viability of the network.

GPRS operates by using the radio channel to transmit short burst containing packets of data. Once a packet is sent, the timeslot used is then available for other GPRS data packets from other users, or for voice calls. In this way a large number of low volume users can be accommodated. To achieve high data rates adjacent timeslots are used, subject to availability.

With a true high speed connectionless data communications link, the possibilities for applications are wide: day-long LAN extension, web browsing, telemetry, multimedia, broadcast services.

\subsection{High Speed Circuit Switched Data (HSCSD)}

HSCSD is a recent (since 1995) effort to quickly provide a capability for high speed data transmission. By extending the current circuit switched data transmission system, it aims to provide data rates of up to $80 \mathrm{kbit} / \mathrm{s}$ without requiring major extensions to the existing GSM system. It will use multiple circuit switched timeslots identical to the current circuit switched data transmission system, sending data over them simultaneously. The aim is to support applications requiring high data rates, such as file transfer, high speed fax, video and multimedia.

The use of multiple timeslots will be restricted to adjacent timeslots. One of the main reasons for this is that GSM handsets presently use a half duplex radio element. Adjacent timeslots allows a half duplex radio element to be used for up to $30 \mathrm{kbit} / \mathrm{s}$. To achieve the highest data rate a handset would require 2 receiver and 1 transmitter radio elements. Handsets supporting the highest data rates will be more expensive than standard GSM handsets because of the 
additional circuitry and the lower volumes of production. Of course this also applies to GPRS handsets which support the highest data rates.

Of particular importance is the ability to set up and tear down circuit switched data calls rapidly, enabling additional timeslots beyond the first to be used in an on demand basis. This should allow HSCSD to share frequencies with voice calls, and hence be available at a reasonable cost to customers. For network operators HSCSD is intended to be able to be implemented without excessive network redesign, and only software changes to the network infrastructure.

The broad HSCSD standard is expected to be finalised by the end of 1996, leading to prototypes in 1997. Nokia displayed a laboratory concept demonstrator at Telecom '95 in Geneva, operating on two timeslots.

One interesting possibility for operators in the Australian environment would be the use of high speed data services in the soon to be released spectrum at $1.8 \mathrm{GHz}$ for DCS1800 systems (GSM at $1800 \mathrm{MHz}$ ). With the greater spectrum likely to be available to each operator, high speed data services could be offered without competing so heavily with voice.

\section{MOBILE DATA AROUND THE WORLD}

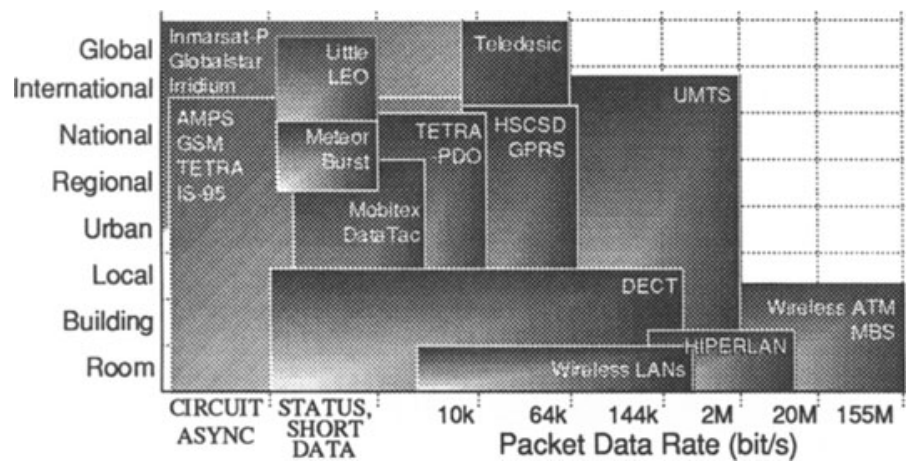

Figure 1. Mobile data comparison chart, contrasting area coverage against data rate.

\subsection{Satellites}

Satellites can be divided into three broad categories: Geostationary (GEO); Low Earth Orbit (LEO) and Little LEO. While the primary satellite focus is on voice, nearly all systems support data communications. Satellite communications systems are not well suited to urban environments because of tall building shadowing.

Geostationary satellite systems (eg. Imarsat) provide broad coverage with a small number of satellites. The distance of the satellite from earth requires the use of large directional antennas and an antenna guidance system if the phone is moving. The maximum data rate supported is $2400 \mathrm{bit} / \mathrm{s}$, and calls are very expensive at around $\mathrm{A} \$ 9$ per minute. A regional spot satellite such as Optus MobilSat charges A\$2.50 per minute. 
LEO systems place a large number of small satellites in close orbit, with communications being handed over from one satellite to the next as they move over the horizon. The closer orbit means lower power transmitters can be used, leading to the expectation of pocket sized handsets. Data rates supported are from 2.4 to $7.2 \mathrm{kbit} / \mathrm{s}$, depending on the system. The proposed Teledesic system has a specific focus on data communications and claims its 840 satellites will support data rates from $16 \mathrm{kbit} / \mathrm{s}$ and higher. (One of Teledesic's backers is Microsoft.) LEO systems aim to charge their calls at a rate similar to cellular phone calls. Launches are planned to begin in 1998.

Little LEOs are store and forward data only systems, usually also incorporating a position location function. By not needing to provide uninterrupted coverage, far fewer satellites need to be launched. ORBCOMM began its launches in 1995, starting with coverage of North America. Target applications include telemetry, tracking, messaging and emergency services.

\subsection{Two way paging}

Two way paging is just beginning to be implemented, mostly as extensions of current paging technology, incorporating a return channel. It is characterised by low data rates (around $1 \mathrm{kbit} / \mathrm{s}$ ), small message size (a few hundred bytes), wide area of coverage and usually a very low speed return path. To be a viable consumer product it must offer lowest cost service, smallest size, excellent coverage and building penetration, and long battery life.

Systems either under trial, or soon to be tested include FLEX, pACT, Nexus and Glenayre. The European paging standard ERMES is also considering adding a return path. The greatest difficulty in such systems is providing enough transmit power from the mobile unit to be received. This means bigger antennae, bigger batteries, multiple receiver aerials, or all three.

\subsection{Low speed}

The Low Speed category includes networks such as DataTAC, MobiTex, Cognito and Paknet. These networks are characterised by low speed data rates (typically under the $10 \mathrm{kbit} / \mathrm{s}$ ), moderate typical message size, moderate to large latency (usually from being a store and forward system), wide area coverage, and typically symmetric uplink and downlink channels.

This category also includes fleet management and vehicular tracking systems such as QuikTRAK, Fleetcomm, Pinpoint, Datatrak and Teletrak. These typically require large aerials for their return transmissions (often via HF radio), have a very low data rates and small packet size. One interesting system, which is wireless though not mobile, is meteor burst communications, where small ( 30 byte) data packets are transmitted up to 1000 miles using VHF radio bounced off the ionosphere.

Low speed systems are usually packet based rather than circuit switched, and are mainly used in specific industrial applications, such as telemetry, EFTPOS, fleet management, stores and inventory, or customised sales applications. The developments in this field are new specialised mobile radio systems (SMR) being introduced with both voice and data capability, such as TETRA, iDEN, Geotek FHMA, and TMR-MAP27.

\subsection{Cellular}

Cellular systems are characterised by moderate data rates, few limits on message size, low latency, cellular coverage (and so high infrastructure cost) and often circuit switched, 
asynchronous connectivity. The existing analogue mobile phone networks AMPS, TACS, and NMT support data rates of $2400 \mathrm{bit} / \mathrm{s}$ reliably, though this can be improved with special protocols in the modems at each end, as mentioned earlier. The digital networks such as GSM, DAMPS and soon IS-95 CDMA support data speeds of around $9.6 \mathrm{kbit} / \mathrm{s}$.

Next generation Personal Communications Systems (PCS) such as PHS, PACS Edge and Ricochet can support higher data rates of $28.8 \mathrm{kbit} / \mathrm{s}$ because of the smaller cell sizes and greater bandwidth allocated. DECT, the new European cordless standard, can support data rates of up to $552 \mathrm{kbit} / \mathrm{s}$ in certain configurations.

The European design for third generation mobile phones, UMTS, calls for data rates inversely related to the coverage area currently occupied. When in microcells within buildings, $2 \mathrm{Mbit} / \mathrm{s}$ would be available. When in medium sized urban cells, $385 \mathrm{kbit} / \mathrm{s}$. In large cells 144 $\mathrm{kbit} / \mathrm{s}$, and in very large cells $16 \mathrm{kbit} / \mathrm{s}$. UMTS has a 2002/2005 timeframe for introduction.

\subsection{High speed}

This category contains the high speed wireless data systems, which typically are wireless LANs. Existing wireless LANs include AIRLAN, WaveLAN and other similar systems. The $2 \mathrm{Mbit} / \mathrm{s}$ raw bit rate provides $600-800 \mathrm{kbit} / \mathrm{s}$ user data rate, which is available to be shared between all the terminals in the area. Range is limited to $50 \mathrm{~m}$ indoors and $300 \mathrm{~m}$ in an optimal outdoors environment. These systems have a capacity for large packets and provide low latency. InfraRed is also a viable option indoors in such limited ranges, and a number of systems are in use.

An increasing use of these systems is to provide point to point links with directional antennas, to provide a LAN link into nearby buildings. In certain cases and where line of sight is available, a range of $10 \mathrm{~km}$ can be achieved. IEEE 802.11 is being developed in an attempt to standardise these systems for both wireless LAN and point to point usage.

In addition to providing a wireless link to the office LAN, these systems have found use in large stores for check-out counters, where not having additional wiring and being able to relocate systems is an advantage.

Future developments primarily concentrate on providing higher data rates, supporting new applications like video, and increasing size of files transferred. The European HIPERLAN standard is being finalised this year, which will support $20 \mathrm{Mbit} / \mathrm{s}$ data with a $50 \mathrm{~m}$ range, or $1 \mathrm{Mbit} / \mathrm{s}$ data with an $800 \mathrm{~m}$ range. It is also expressly designed to support slowly moving $(36 \mathrm{~km} / \mathrm{h})$ terminals. The Japanese are developing a similar standard supporting $10 \mathrm{Mbit} / \mathrm{s}$, and there are North American proposals to build on HIPERLAN to develop a local standard (Apple's NII and WinForum's SUPERNET, which have recently combined).

Wireless versions of ATM are being considered and proposed in a number of forms. Certainly any future wireless LAN system will have ATM connection capability. Olivetti are testing in laboratory a system using ATM packets sent over the air. The goal for such systems is to be able to support broadband applications such as HDTV (6Mbit/s). Mobile Broadband System (MBS) is a European project, complimentary to UMTS in telephony, to support data rates from $2 \mathrm{Mbit} / \mathrm{s}$ up to $155 \mathrm{Mbit} / \mathrm{s}$ (B-ISDN compatible). Since it is planned to operate in the $60 \mathrm{GHz}$ frequency range, it will be several years before the equipment is available and affordable. 


\section{CONCLUSION}

Any new wireless system being designed will support data communications to meet the demand of the growing market. Indeed, current wireless systems in many cases are enhancing their current support for data communications. GSM in particular will be enhanced in ways which will allow the widespread use of data communications, with improved performance and more attractive costs. However, the limitations which wireless imposes still call for innovative software design and careful systems integration - and convergence between telecommunications and computing.

\section{REFERENCES}

Alanko T., Kojo M., Laamanen I., Liljeberg M., Moilanen M., Raatikainen K. (1994) Measured Performance of Data Transmission Over Cellular Telephone Networks. University of Helsinki Department of Computer Science, Report C-1994-53.

Chambers, P.(ed.) (April 8th, 1996) Wireless LAN Market forecasts. Wireless Office Newsletter, Telecomeuropa.

Data Communications (March 21, 1995) Special Report / Wireless Data Services. Data Communications.

ETSI SMG2 and SMG3 GPRS working groups. (1994-96) GSM 02.60, GSM 03.60, GSM 04.60 and associated working documents. ETSI.

GSM 04.22 (1992) Radio Link Protocol (RLP) for data and telematic services on the MS-BSS and BSS-MSC interface. ETSI.

Gilchrist, P. (1996) One stop data shop FROM GSM's GPRS. Mobile Communications International, Issue 29, 62-64.

Hämäläinen, J. (1996) GSM's support of HIGH SPEED DATA. Mobile Communications International, Issue 27, 72-78.

Mouley, M. and Pautet, B. (1993) The GSM System for Mobile Communications. Published by the authors, ISBN 2-9507190-0-7.

Pryor, R. (1996) DECT - From Cordless to Wireless to Tetherless. Proceedings of Mobiles '96, IIR Conferences

Rune, T. (1995) Wireless Local Area Networks. Proceedings of Telecom ‘95, ITU Geneva. Yankee Group (April 1996) Sending data through the air, d.Comm. The Economist.

\section{BIOGRAPHY}

John Leske graduated from the University of Adelaide in 1987 with an Honours degree in Electrical and Electronic Engineering. He worked for a number of years in signal processing and high speed computer design before heading off to see why his ancestors had left Europe. After designing safety systems for oil platforms in the UK, he decided that despite there being some very nice places in Europe, perhaps some of his ancestor's reasons were valid. He joined CTIN in 1994 as a Research Engineer, concentrating on mobile data. 